\title{
Linear transmitter with correlated noises
}

\author{
P. F. Góra \\ M. Smoluchowski Institute of Physics and Complex Systems Research Center, Jagellonian \\ University, Reymonta 4, 30-059 Kraków, Poland
}

\begin{abstract}
A linear transmitter with correlated Gaussian white additive and multiplicative noises and a periodic signal coupled either additively or multiplicatively is considered. The correlations have a weak destructive effect in case of the additive signal and a strong constructive effect in case of the multiplicative one. Analytical results for the linear transmitter with the additive signal agree with those obtained previously by a different approach. We also show how to analytically calculate certain expectation values involving exponentials of Gaussian processes.
\end{abstract}

Key words: Correlated Gaussian noises, stochastic resonance, signal-to-noise ratio PACS: 05.40.Ca

\section{Introduction}

The fact that noise can play a constructive role in many physical systems is now widely recognized. The best-known examples of such phenomena are the stochastic resonance (SR) [1] and Brownian ratchets [2]. SR is a phenomenon in which the response of a dynamical system is optimized by the presence of a specific level of noise and has been detected in so many seemingly different systems that it has been claimed to be "an inherent property of rate-modulated series of events" [3]. However, it has been recently suggested that the functioning of important natural devices, e.g., communication and information processing in neural systems or subthreshold signal detection in biological receptors, rely on phase synchronization rather than stochastic resonance [4]. The SR is usually measured by the signal-tonoise ratio (SNR) for the output signal but new measures are being proposed and discussed $[4,5,6]$.

Email address: gora@if.uj.edu.pl (P. F. Góra). 
One of the issues that is now debated and that is an origin of many unexpected physical phenomena, is the role of correlations between various noises present in the system. This point has been particularly stressed in research on Duffing oscillator with additive and multiplicative noises $[7,8]$, where correlations are responsible for huge changes in the activation rate, on Brownian ratchets [9], where correlations between the additive and multiplicative noises can lead to current reversal, and on a nonlinear rotator [10], where full correlations between two noises amount to nullifying one of them, leaving one of the states effectively noise-free and allowing for the passage of incoming signals of vanishing intensity. Other contexts in which correlations between various noise terms appear to be important include a quantum dimer under the influence of colored noises [11], a coupled neuron network [12], synchronization of chaotic oscillators [13], detecting the gravitational background [14], and medical imaging [15]. The effect of correlations between a Gaussian white noise and a Gaussian colored noise has been discussed in Ref. [16].

It is sometimes very difficult to find analytical results for nonlinear models and it is for that reason that models which admit rigorous solutions are very interesting. One of such models is that of an overdamped linear transmitter, which in full form reads

$$
\dot{a}=\left(f_{1}(t)+f_{2}(t) \xi_{1}(t)\right) a+f_{3}(t)+f_{4}(t) \xi_{2}(t)
$$

where $\xi_{1,2}(t)$ are the noise terms. It should be mentioned that while (1) formally leads to a linear equation of motion with time-dependent coefficients, a multiplicative coupling between the noise and the system means a "hidden" nonlinearity: The noise is supposed to represent many unobserved degrees of freedom coupled to the transmitting process in a nonlinear manner. Although chemical reaction with a fluctuating barrier provided the original motivation for this model, with $a$ being the concentration of a reagent, the presence of the additive noise, representing the thermal bath acting on the system, and an additive deterministic signal possibly lead to realizations that admit negative values of $a(t)$. It, therefore, can no longer be regarded as the concentration of a chemical species. Nevertheless, systems of this type can still describe processes of a paramount physical interest like the electric potential across a molecular membrane [17] or provide a "skeletal" model of various enzymatic reactions [18]. This model, in less general forms, has also been discussed in Refs. [19]. With one exception (see below), possible implications of the presence of correlations between the two noise terms in (1) have not been addressed so far. Including such correlations is an obvious extension to the existing research on models belonging to the general class (1) and is the subject of the present paper.

There is one important exception to the general statement made in the preceding paragraph. Berdichevsky and Gitterman in Ref. [20] considered the linear transmitter with correlated noises and an additively coupled signal. In order to calculate the 
correlation function of the process $a(t)$ they obtained a whole hierarchy $\langle a\rangle,\langle\xi a\rangle$, $\left\langle\xi^{2} a\right\rangle$ etc, where $\xi$ is a noise term, and needed a procedure to close the hierarchy. They resorted to considering asymmetric, dichotomous, exponentially correlated noises and obtained results for Gaussian white noises only as a limiting case. In this paper we show how to treat the Gaussian case directly; we think this approach is important and interesting, even though the behavior predicted in Ref. [20] is certainly more "rich" than that for a pure Gaussian white noise. We also slightly differ with the authors of that reference in our analysis of final results. In addition, we extend our previous discussion of a model with a multiplicative signal [18] to the case of correlated noises. We find that correlations play an important role in this system.

This paper is organized as follows: In Section 2 we introduce the model and discuss a couple of its formal features. We give analytical results for the expectation value, the correlation function, and the power spectrum of the system with an additive noise in Section 3, and analytical and numerical results for the system with a signal coupled to the system multiplicatively (parametrically) in Section 4. A short discussion in Section 5 follows. Mathematical details are presented in the Appendix; although delegated to an Appendix, these details represent an important part of this paper.

\section{The model}

Consider a system described by the following equation:

$$
\dot{a}=-\left(k_{a}+\kappa \xi_{1}(t)+f(t)\right) a+\lambda \xi_{2}(t)+g(t) .
$$

$\xi_{1}(t), \xi_{2}(t)$ represent the multiplicative and additive noises, respectively, acting on the system. $f(t)$ and $g(t)$ are deterministic external stimulations; in the present paper we assume that either one of them vanishes identically. The constants $\kappa$ and $\lambda$ allow for an independent manipulation of the noises' strengths. The model (2) belongs to the general class of (1) but is simplified to keep only its most important features. The multiplicative noise represents fluctuations of a barrier and the additive noise is a thermal bath acting on the system.

The noises acting on the system need not to be independent. On the contrary, it is very likely that both the thermal bath and the process affecting the barrier height are correlated, as the molecular mechanisms responsible for these noises are not independent. It is the objective of the present paper to examine the effect of this correlation on the dynamics of the system. To keep the model as simple as possible, we assume that the noises $\xi_{1}(t), \xi_{2}(t)$ form a two dimensional Gaussian process, 
point-correlated in time:

$$
\left\langle\left[\begin{array}{l}
\xi_{1}(t) \\
\xi_{2}(t)
\end{array}\right]\left[\xi_{1}\left(t^{\prime}\right) \xi_{2}\left(t^{\prime}\right)\right]\right\rangle=\delta\left(t-t^{\prime}\right)\left[\begin{array}{ll}
1 & c \\
c & 1
\end{array}\right],
$$

where $c \in[-1,1]$ is the correlation coefficient. We now perform the Cholesky decomposition [21] of the matrix in (3) and use it to construct the noises from two independent processes:

$$
\left[\begin{array}{l}
\xi_{1}(t) \\
\xi_{2}(t)
\end{array}\right]=\left[\begin{array}{cc}
1 & 0 \\
c & \sqrt{1-c^{2}}
\end{array}\right]\left[\begin{array}{l}
\xi(t) \\
\eta(t)
\end{array}\right] .
$$

Here $\xi(t), \eta(t)$ are two uncorrelated Gaussian white noises $(\mathrm{GWN}):\langle\xi(t)\rangle=0$, $\langle\eta(t)\rangle=0,\left\langle\xi(t) \xi\left(t^{\prime}\right)\right\rangle=\left\langle\eta(t) \eta\left(t^{\prime}\right)\right\rangle=\delta\left(t-t^{\prime}\right),\left\langle\xi(t) \eta\left(t^{\prime}\right)\right\rangle=0$, and all higher correlations factorize. A more general mechanism of describing correlations between the noises has been introduced in Ref. [7], but we will show that the dynamics of the system does not significantly depend on the detailed mechanism of introducing the correlations.

We further assume that the external stimulations have a simple periodic form. The evolution equation (2) thus becomes

$\dot{a}=-\left(k_{a}+\kappa \xi(t)+A_{\mathrm{m}} \cos (\Omega t+\phi)\right) a+\lambda c \xi(t)+\lambda \sqrt{1-c^{2}} \eta(t)+A_{\mathrm{a}} \cos (\Omega t+\phi)$,

where $\phi$ is an initial phase of the signal. According to what we have said above, only one of the amplitudes $A_{\mathrm{m}}, A_{\mathrm{a}}$ does not vanish.

The equation (5) can be formally solved. This solution can be used to calculate its expectation value and the autocorrelation function. Before we do that, we must first solve a couple of problems that will appear during these calculations.

First, these calculations involve certain expectation values that, to our best knowledge, have not been calculated so far: One has the form $\left\langle\xi(t) e^{-\int_{t}^{T} p\left(t^{\prime}\right) \xi\left(t^{\prime}\right) d t^{\prime}}\right\rangle$ and the other $\left\langle\xi\left(t_{1}\right) \xi\left(t_{2}\right) e^{-\int_{t}^{T} q\left(t^{\prime}\right) \xi\left(t^{\prime}\right) d t^{\prime}}\right\rangle$, where $p(\cdot), q(\cdot)$ are certain deterministic functions and $\xi(\cdot)$ is a GWN. We show how to calculate them in the Appendix.

Second, the correlation functions should be averaged over the initial phase of the signals, as otherwise they will not be stationary $[17,22,23]$. Therefore, we will use 
correlation functions of the form

$$
\left\langle\left\langle a\left(t_{1}\right) a\left(t_{2}\right)\right\rangle\right\rangle=\frac{1}{2 \pi} \int_{0}^{2 \pi}\left\langle a\left(t_{1}\right) a\left(t_{2}\right)\right\rangle d \phi .
$$

The braces $\langle\cdots\rangle$ on the right-hand side of Eq. (6) stand for the average over realizations of the noises.

Finally, calculating the power spectra of the processes described by Eq. (5) is our ultimate goal. Let $a(t)$ be a solution to the equation (5). We expect that it becomes stationary for $t \rightarrow \infty$. The simplest estimate of the power spectrum is the square modulus of the Fourier transform of the process. If we were to calculate the power spectrum numerically, we would calculate a particular realization of $a(t)$, reject several (perhaps many) initial values until the series becomes stationary, calculate the power spectrum of the remaining series and finally average over the realization of the noises and the initial phase of the signal. We now follow this programme using the analytical, not a numerical, solution. Thus, for the power spectrum we obtain

$$
\begin{aligned}
P(\omega) & =\lim _{T \rightarrow \infty} \frac{1}{2 T} \lim _{T_{0} \rightarrow \infty}\left\langle\left\langle\left|\int_{T_{0}-T}^{T_{0}+T} a(t) e^{i \omega t} d t\right|^{2}\right\rangle\right\rangle \\
& =\lim _{T \rightarrow \infty} \frac{1}{2 T} \lim _{T_{0} \rightarrow \infty} \int_{T_{0}-T}^{T_{0}+T} d t_{1} \int_{T_{0}-T}^{T_{0}+T} d t_{2}\left\langle\left\langle a\left(t_{1}\right) a\left(t_{2}\right)\right\rangle\right\rangle e^{i \omega\left(t_{1}-t_{2}\right)} \\
& =\lim _{T \rightarrow \infty} \frac{1}{2 T} \lim _{T_{0} \rightarrow \infty} \int_{-T}^{T} d t_{1} \int_{-T}^{T} d t_{2}\left\langle\left\langle a\left(T_{0}+t_{1}\right) a\left(T_{0}+t_{2}\right)\right\rangle\right\rangle \cos \omega\left(t_{1}-t_{2}\right) .
\end{aligned}
$$

Note that in finite times approximants of the right-hand side of Eq. (7) we must always have $T_{0} \gg T \gg 0$ and the limit $T_{0} \rightarrow \infty$ must be taken first. After a simple change of variables, we obtain

$$
\begin{aligned}
P(\omega)= & \lim _{T \rightarrow \infty} \frac{1}{2 T} \int_{0}^{2 T} d \tau \cos \omega \tau \times \\
& \lim _{T_{0} \rightarrow \infty} \int_{\tau-2 T}^{\tau+2 T} d t\left\langle\left\langle a\left(T_{0}+\frac{1}{2} t-\frac{1}{2} \tau\right) a\left(T_{0}+\frac{1}{2} t+\frac{1}{2} \tau\right)\right\rangle\right\rangle .
\end{aligned}
$$

If the process $a(t)$ becomes stationary in the asymptotic regime, its autocorrelation 
function depends only on the difference of its arguments:

$$
\lim _{t \rightarrow \infty}\left\langle\left\langle a\left(t-\frac{1}{2} \tau\right) a\left(t+\frac{1}{2} \tau\right)\right\rangle\right\rangle=C(\tau)
$$

We thus have

$$
P(\omega)=2 \int_{0}^{\infty} \cos \omega \tau C(\tau) d \tau
$$

If the process $a(t)$ is stationary, we immediately have

$$
C(\tau)=\lim _{t \rightarrow \infty}\langle\langle a(t-\tau) a(t)\rangle\rangle=\lim _{t \rightarrow \infty}\langle\langle a(t) a(t+\tau)\rangle\rangle
$$

and we can see that Eq. (10) is in the standard Wiener-Khinchin form.

\section{An additive signal}

We now turn to discussing specific forms of the general system (5). We start with a system with an additive periodic signal, described by the following equation:

$$
\dot{a}=-\left(k_{a}+\kappa \xi(t)\right) a+\lambda c \xi(t)+\lambda \sqrt{1-c^{2}} \eta(t)+A \cos (\Omega t+\phi) .
$$

The equation (12) has a formal solution

$$
\begin{aligned}
a(t)= & e^{-k_{a} t} \exp \left[-\kappa \int_{0}^{t} \xi\left(t^{\prime}\right) d t^{\prime}\right] a_{0} \\
& +\lambda \int_{0}^{t} e^{-k_{a}\left(t-t^{\prime}\right)} \exp \left[-\kappa \int_{t^{\prime}}^{t} \xi\left(t^{\prime \prime}\right) d t^{\prime \prime}\right]\left(c \xi\left(t^{\prime}\right)+\sqrt{1-c^{2}} \eta\left(t^{\prime}\right)\right) d t^{\prime} \\
& +A \int_{0}^{t} e^{-k_{a}\left(t-t^{\prime}\right)} \exp \left[-\kappa \int_{t^{\prime}}^{t} \xi\left(t^{\prime \prime}\right) d t^{\prime \prime}\right] \cos \left(\Omega t^{\prime}+\phi\right) d t^{\prime}
\end{aligned}
$$

where $a_{0}$ is the initial value of the process. For simplicity, we assume in what follows that $a_{0} \equiv 0$.

For the expectation value of the process we obtain 


$$
\begin{aligned}
\langle a(t)\rangle & =\frac{A \cos (\Omega t+\phi+\widetilde{\phi})}{\sqrt{\left(k_{a}-\frac{1}{2} \kappa^{2}\right)^{2}+\Omega^{2}}}-\frac{\lambda \kappa c}{2\left(k_{a}-\frac{1}{2} \kappa^{2}\right)} \\
& +e^{-\left(k_{a}-\frac{1}{2} \kappa^{2}\right) t}\left(\frac{\lambda \kappa c}{2\left(k_{a}-\frac{1}{2} \kappa^{2}\right)}-\frac{A \cos (\phi+\widetilde{\phi})}{\sqrt{\left(k_{a}-\frac{1}{2} \kappa^{2}\right)^{2}+\Omega^{2}}}\right)
\end{aligned}
$$

where $\tan \widetilde{\phi}=-\Omega /\left(k_{a}-\frac{1}{2} \kappa^{2}\right)$.

As we can see, only the part of the additive noise that is correlated with the multiplicative noise contributes to the expectation value (14). There is a maximal admissible level of the multiplicative noise, $\kappa_{\max }=\sqrt{2 k_{a}}$, beyond which the process diverges. In the asymptotic regime, $t \rightarrow \infty$, the system displays stationary oscillations. The amplitude of these oscillations grows monotonically as the multiplicative noise increases from zero to the maximal level. This is a manifestation of a constructive role of the noise and it is essentially the same as in the case without the additive noise [17]. There is one important difference, though: Without the correlated additive noise, $\langle a(t)\rangle$ oscillates around zero, and with this noise present, it oscillates around a value that depends on the correlation level and strengths of both the multiplicative and additive noises. If we now calculate the amplitude of the oscillations relative to this flat background,

$$
\frac{\text { oscillations amplitude }}{\text { background }}=\frac{A}{\lambda \kappa|c|} \cdot \frac{2\left(k_{a}-\frac{1}{2} \kappa^{2}\right)}{\sqrt{\left(k_{a}-\frac{1}{2} \kappa^{2}\right)^{2}+\Omega^{2}}},
$$

we can see that the relative amplitude formally diverges for $\lambda \kappa c \rightarrow 0$ (there is no threshold and the system can transfer the periodic signal even in the absence of the noise) and monotonically decreases to zero as the level of the multiplicative noise increases towards its maximal value. In other words, if the strength of the multiplicative noise is too large, the periodic stimulation gets drowned in the flat background. In this respect correlations between the multiplicative and additive noises act destructively on the system.

If we take an average not only over realizations of the noises but also over the phase of the incoming signal, all oscillations vanish:

$$
\langle\langle a(t)\rangle\rangle \underset{t \rightarrow \infty}{\longrightarrow} a_{\infty}=-\frac{\lambda \kappa c}{2\left(k_{a}-\frac{1}{2} \kappa^{2}\right)}
$$

We now turn to calculating the correlations for the process $a(t)$. The full expressions are rather long, and therefore we present the results in the asymptotic $(t \rightarrow$ $\infty)$ regime only and after averaging on both the realizations of the noises and the initial phase: 


$$
\begin{gathered}
\left\langle\left\langle a\left(t-\frac{1}{2} \tau\right) a\left(t+\frac{1}{2} \tau\right)\right\rangle\right\rangle \underset{t \rightarrow \infty}{\longrightarrow}\left(\frac{\lambda \kappa c}{2\left(k_{a}-\frac{1}{2} \kappa^{2}\right)}\right)^{2}+\frac{A^{2} \cos \Omega \tau}{2\left(\left(k_{a}-\frac{1}{2} \kappa^{2}\right)^{2}+\Omega^{2}\right)}+ \\
\left(\frac{A^{2} \kappa^{2}}{4\left(k_{a}-\kappa^{2}\right)\left(\left(k_{a}-\frac{1}{2} \kappa^{2}\right)^{2}+\Omega^{2}\right)}+\frac{\lambda^{2}}{2\left(k_{a}-\kappa^{2}\right)}+\frac{(\lambda \kappa c)^{2}\left(k_{a}-\frac{1}{4} \kappa^{2}\right)}{2\left(k_{a}-\kappa^{2}\right)\left(k_{a}-\frac{1}{2} \kappa^{2}\right)^{2}}\right) e^{-\left(k_{a}-\frac{1}{2} \kappa^{2}\right) \tau}
\end{gathered}
$$

We can see that the correlation function (17) exists only if the multiplicative noise level satisfies $\kappa<\sqrt{k_{a}}=\kappa_{\max } / \sqrt{2}$. For the multiplicative noise levels in the range $\kappa_{\max } / \sqrt{2} \leq \kappa<\kappa_{\max }$, the first moment of the process $a(t)$ converges, but the second does not.

The first term in (17) corresponds to the constant shift introduced by this part of the additive noise that is correlated to the multiplicative one; it is equal to $a_{\infty}^{2}$. The second describes the oscillations in the correlation function introduced by the external periodic forcing in (5). The remaining terms describe the diffusive background: First of them is present even in the absence of the additive noise. The second contains contributions from both the correlated and uncorrelated parts of the additive noise, and only the correlated part contributes to the remaining one. Note that the sign of this term is always positive: the presence of correlations raises the diffusive background. The correlations act destructively on the system with a additive signal.

The correlation function (17), leads to, via Eq. (10), to the following power spectrum:

$$
P(\omega)=P_{0} \delta(\omega)+P_{\text {signal }}(\delta(\omega-\Omega)+\delta(\omega+\Omega))+P_{\text {back }}(\omega),
$$

where

$$
P_{0}=\left(\frac{\lambda \kappa c}{2\left(k_{a}-\frac{1}{2} \kappa^{2}\right)}\right)^{2}
$$

is the power density introduced at zero frequency by the constant shift,

$$
P_{\text {signal }}=\frac{A^{2}}{\left(k_{a}-\frac{1}{2} \kappa^{2}\right)^{2}+\Omega^{2}}
$$

is the power density associated with the oscillatory term, and 


$$
\begin{gathered}
P_{\text {back }}(\omega)= \\
\frac{A^{2} \kappa^{2}\left(k_{a}-\frac{1}{2} \kappa^{2}\right)^{2}+\lambda^{2}\left[2\left(k_{a}-\frac{1}{2} \kappa^{2}\right)^{2}+c^{2} \kappa^{2}\left(k_{a}-\frac{1}{4} \kappa^{2}\right)\right]\left[\left(k_{a}-\frac{1}{2} \kappa^{2}\right)^{2}+\Omega^{2}\right]}{4\left(k_{a}-\kappa^{2}\right)\left(k_{a}-\frac{1}{2} \kappa^{2}\right)\left[\left(k_{a}-\frac{1}{2} \kappa^{2}\right)^{2}+\Omega^{2}\right]\left[\left(k_{a}-\frac{1}{2} \kappa^{2}\right)^{2}+\omega^{2}\right]}
\end{gathered}
$$

is the power density associated with the diffusive background. We can now calculate the signal-to-noise ratio (SNR) as the ratio between the power densities transferred by the signal and by the diffusive background at the frequency of the signal:

$$
\begin{gathered}
\operatorname{SNR}\left(\kappa^{2}, Z, c\right)=\frac{P_{\text {signal }}}{P_{\text {back }}(\omega= \pm \Omega)}= \\
\frac{\left(k_{a}-\kappa^{2}\right)\left(k_{a}-\frac{1}{2} \kappa^{2}\right)\left[\left(k_{a}-\frac{1}{2} \kappa^{2}\right)^{2}+\Omega^{2}\right]}{\kappa^{2}\left(k_{a}-\frac{1}{2} \kappa^{2}\right)^{2}+Z\left[\left(k_{a}-\frac{1}{2} \kappa^{2}\right)^{2}+\Omega^{2}\right]\left[2\left(k_{a}-\frac{1}{2} \kappa^{2}\right)^{2}+c^{2} \kappa^{2}\left(k_{a}-\frac{1}{4} \kappa^{2}\right)\right]}
\end{gathered}
$$

where $Z=(\lambda / A)^{2}$ measures the relative strength of the additive noise and the signal. Regardless of the values of other parameters, SNR drops to zero for $\kappa^{2}=k_{a}$, meaning that the total power density comes from the noise and the signal is totally undistinguishable from it. For $Z=0$ (20) formally diverges for $\kappa \rightarrow 0$ (recall that since there is no threshold, the system transfers the signal even for a vanishing multiplicative noise). SNR remains finite for $Z>0$ and since the second term in the denominator of (20) is nonnegative, $\operatorname{SNR}\left(\kappa^{2}, Z, c\right) \leqslant \operatorname{SNR}\left(\kappa^{2}, 0, \cdot\right)$. If the above inequality is strong, the presence of the additive noise acts destructively on the system's capabilities to transfer the signal. In any case, SNR decreases monotonically as the multiplicative noise increases: there is no stochastic resonance.

For weak signals $(Z \geqslant 1)$ the SNR scales as $Z^{-1}$, which is readily seen in (20). Furthermore, $\forall Z>0, \kappa: \operatorname{SNR}\left(\kappa^{2}, Z, c \neq 0\right)<\operatorname{SNR}\left(\kappa^{2}, Z, 0\right)$ : If the additive and multiplicative noises are correlated, the system's capabilities to transfers the periodic signal are slightly worse than those of the system with uncorrelated noises. Numerical simulations show that the system can transfer signals as weak as $Z \lesssim$ 100 , but for such a weak signal the difference between the $c=0$ and $|c|=1$ cases is scarcely noticeable.

Finally, we may ask what is the relative "height" of the $\delta$-peak in the spectrum associated with the signal to the $\delta$-peak associated with what manifests itself as a constant forcing and results from the presence of the correlations. Not surpris- 
ingly,

$$
\frac{P_{\text {signal }}}{P_{0}}=\frac{A^{2}}{(\lambda \kappa c)^{2}} \cdot \frac{4\left(k_{a}-\frac{1}{2} \kappa^{2}\right)^{2}}{\left(k_{a}-\frac{1}{2} \kappa^{2}\right)^{2}+\Omega^{2}},
$$

which once again shows that in the presence of the correlations between the multiplicative and additive noises, the signal is drowned by the correlated noise as the multiplicative noise level increases. Note that the ratio (21) diverges if $\lambda \kappa c=0$; this reflects the fact that if any of the noises vanishes, or if the noises are uncorrelated, there is no constant shift in the output, cf. Eq. (14) above.

\section{A multiplicative signal}

We now consider a model described by the following equation:

$$
\dot{a}=-\left(k_{a}+\kappa \xi(t)+A \cos (\Omega t+\phi)\right) a+\lambda c \xi(t)+\lambda \sqrt{1-c^{2}} \eta(t),
$$

where all quantities have meanings and properties of the corresponding quantities in Eq. (12) above. The only difference is that the signal is now coupled parametrically to the transmitter. We have discussed this model in Ref. [18], but only for the uncorrelated case $(c=0)$, where we have shown that because of the presence of the additive noise, the system responds, in terms of its autocorrelation function, to the signal even though the average realization of $a(t)$ goes to zero.

\subsection{Analytical results}

A formal solution to Eq. (22) reads

$$
\begin{gathered}
a(t)=\lambda \int_{0}^{t} e^{-k_{a}\left(t-t^{\prime}\right)} \exp \left[-\kappa \int_{t^{\prime}}^{t} \xi\left(t^{\prime \prime}\right) d t^{\prime \prime}-A \int_{t^{\prime}}^{t} \cos \left(\Omega t^{\prime \prime}+\phi\right) d t^{\prime \prime}\right] \times \\
\left(c \xi\left(t^{\prime}\right)+\sqrt{1-c^{2}} \eta\left(t^{\prime}\right)\right) d t^{\prime}
\end{gathered}
$$

where we have assumed that the initial value $a(0)=0$ for simplicity. As in the previous Section, we will use this formal solution in calculating the expectation value of the process $a(t)$ and its autocorrelation function. The calculations run similarly to those for the additive noise and make use of the expectation values evaluated in the Appendix. For the expectation value we obtain 


$$
\begin{aligned}
\langle a(t)\rangle=- & \frac{1}{2} \lambda \kappa c \int_{0}^{t} e^{-\left(k_{a}-\frac{1}{2} \kappa^{2}\right)\left(t-t^{\prime}\right)} \times \\
& \quad \exp \left[-\frac{2 A}{\Omega} \sin \frac{1}{2} \Omega\left(t-t^{\prime}\right) \cos \left(\phi+\frac{1}{2} \Omega\left(t+t^{\prime}\right)\right)\right] d t^{\prime} .
\end{aligned}
$$

If the signal is weak, $|A / \Omega| \ll 1$, we may expand the expectation value (24), not averaged over the initial phase, to obtain

$$
\begin{aligned}
& \langle a(t)\rangle \underset{t \rightarrow \infty}{\longrightarrow}-\frac{c \lambda \kappa}{2\left(k_{a}-\frac{1}{2} \kappa^{2}\right)}\left(1+\frac{A}{\Omega} \frac{\cos (\Omega t+\phi+\widetilde{\phi})}{\sqrt{1+\tan ^{2} \widetilde{\phi}}}\right), \\
& \tan \widetilde{\phi}=\frac{k_{a}-\frac{1}{2} \kappa^{2}}{\Omega} .
\end{aligned}
$$

We can see that in the limit of weak signals, the transmitter asymptotically displays a constant shift and oscillations. The amplitude of these oscillations relative to the constant background grows steadily as the multiplicative noise level increases towards $\kappa_{\max }$ : The oscillations induced by a parametrically coupled signal are not drowned in the noisy background. The presence of these oscillations is clearly a constructive effect of the correlations as in the uncorrelated case, $c=0,\langle a(t)\rangle \equiv 0$.

If we further average Eq. (24) over the phase of the incoming signal, we obtain

$$
\begin{aligned}
\langle\langle a(t)\rangle\rangle=-\frac{1}{2} \lambda \kappa c \int_{0}^{t} d t^{\prime} e^{-\left(k_{a}-\frac{1}{2} \kappa^{2}\right)\left(t-t^{\prime}\right)} \times \\
\quad \frac{1}{2 \pi} \int_{0}^{2 \pi} d \phi \exp \left[-\frac{2 A}{\Omega} \sin \frac{1}{2} \Omega\left(t-t^{\prime}\right) \cos \left(\phi+\frac{1}{2} \Omega\left(t+t^{\prime}\right)\right)\right] \\
=-\frac{1}{2} \lambda \kappa c \int_{0}^{t} d t^{\prime} e^{-\left(k_{a}-\frac{1}{2} \kappa^{2}\right) t^{\prime}} \frac{1}{2 \pi} \int_{0}^{2 \pi} d \phi \exp \left[-\frac{2 A}{\Omega} \sin \frac{1}{2} \Omega t^{\prime} \cos \phi\right],
\end{aligned}
$$

since the integration over $\phi$ runs over the entire period of the integrand and therefore the value of the inner integral cannot depend on $\frac{1}{2} \Omega\left(t+t^{\prime}\right)$. An integral representation of the modified Bessel function

$$
I_{0}(z)=\sum_{n=0}^{\infty} \frac{(z / 2)^{2 n}}{(n !)^{2}}=\frac{1}{2 \pi} \int_{0}^{2 \pi} \exp (z \cos \phi) d \phi
$$


can immediately be recognized [24]:

$$
\langle\langle a(t)\rangle\rangle=-\frac{1}{2} c \lambda \kappa \int_{0}^{t} e^{-\left(k_{a}-\frac{1}{2} \kappa^{2}\right) t^{\prime}} I_{0}\left(\frac{2 A}{\Omega} \sin \frac{1}{2} \Omega t^{\prime}\right) d t^{\prime} .
$$

Furthermore, as

$$
\begin{gathered}
0 \leqslant \int_{t}^{\infty} e^{-\left(k_{a}-\frac{1}{2} \kappa^{2}\right) t^{\prime}} I_{0}\left(\frac{2 A}{\Omega} \sin \frac{1}{2} \Omega t^{\prime}\right) d t^{\prime} \leqslant \int_{t}^{\infty} e^{-\left(k_{a}-\frac{1}{2} \kappa^{2}\right) t^{\prime}} I_{0}\left(\frac{2 A}{\Omega}\right) d t^{\prime} \\
=\frac{1}{k_{a}-\frac{1}{2} \kappa^{2}} I_{0}\left(\frac{2 A}{\Omega}\right) e^{-\left(k_{a}-\frac{1}{2} \kappa^{2}\right) t} \underset{t \rightarrow \infty}{\longrightarrow} 0
\end{gathered}
$$

we may conclude that

$$
\langle\langle a(t)\rangle\rangle \underset{t \rightarrow \infty}{\longrightarrow}-\frac{1}{2} \lambda \kappa c \int_{0}^{\infty} e^{-\left(k_{a}-\frac{1}{2} \kappa^{2}\right) t^{\prime}} I_{0}\left(\frac{2 A}{\Omega} \sin \frac{1}{2} \Omega t^{\prime}\right) d t^{\prime}
$$

provided that $\kappa<\kappa_{\max }=\sqrt{2 k_{a}}$; otherwise the process is divergent, which is readily seen directly from Eq. (24). Observe that if $A \geqslant k_{a}-\frac{1}{2} \kappa^{2}$, the expectation value (30) formally diverges in the limit $\Omega \rightarrow 0$, even though realizations for some particular values of the initial phase $\phi$ may be convergent.

For the correlation function we obtain

$$
\left\langle\left\langle a\left(t-\frac{1}{2} \tau\right) a\left(t+\frac{1}{2} \tau\right)\right\rangle\right\rangle=J_{1}+J_{2}+J_{3},
$$

where

$$
\begin{aligned}
& J_{1}=\lambda^{2} e^{-\left(k_{a}-\frac{1}{2} \kappa^{2}\right) \tau} \int_{0}^{t} d s e^{-2\left(k_{a}-\kappa^{2}\right) s} I_{0}\left(\frac{A}{\Omega} Z_{1}\right) \\
& J_{2}=\frac{3}{2}(\lambda \kappa c)^{2} e^{-\left(k_{a}-\frac{1}{2} \kappa^{2}\right) \tau} \int_{0}^{t-\frac{1}{2} \tau} d s e^{-2\left(k_{a}-\kappa^{2}\right) s} \int_{0}^{t-\frac{1}{2} \tau-s} d u e^{-\left(k_{a}-\frac{1}{2} \kappa^{2}\right) u} I_{0}\left(\frac{A}{\Omega} Z_{2}\right) \\
& J_{3}=\frac{1}{4}(\lambda \kappa c)^{2} \int_{0}^{t-\frac{1}{2} \tau} d s e^{-\left(k_{a}-\frac{1}{2} \kappa^{2}\right) s} \int_{0}^{\tau} d u e^{-\left(k_{a}-\frac{1}{2} \kappa^{2}\right) u} I_{0}\left(\frac{A}{\Omega} Z_{3}\right)
\end{aligned}
$$



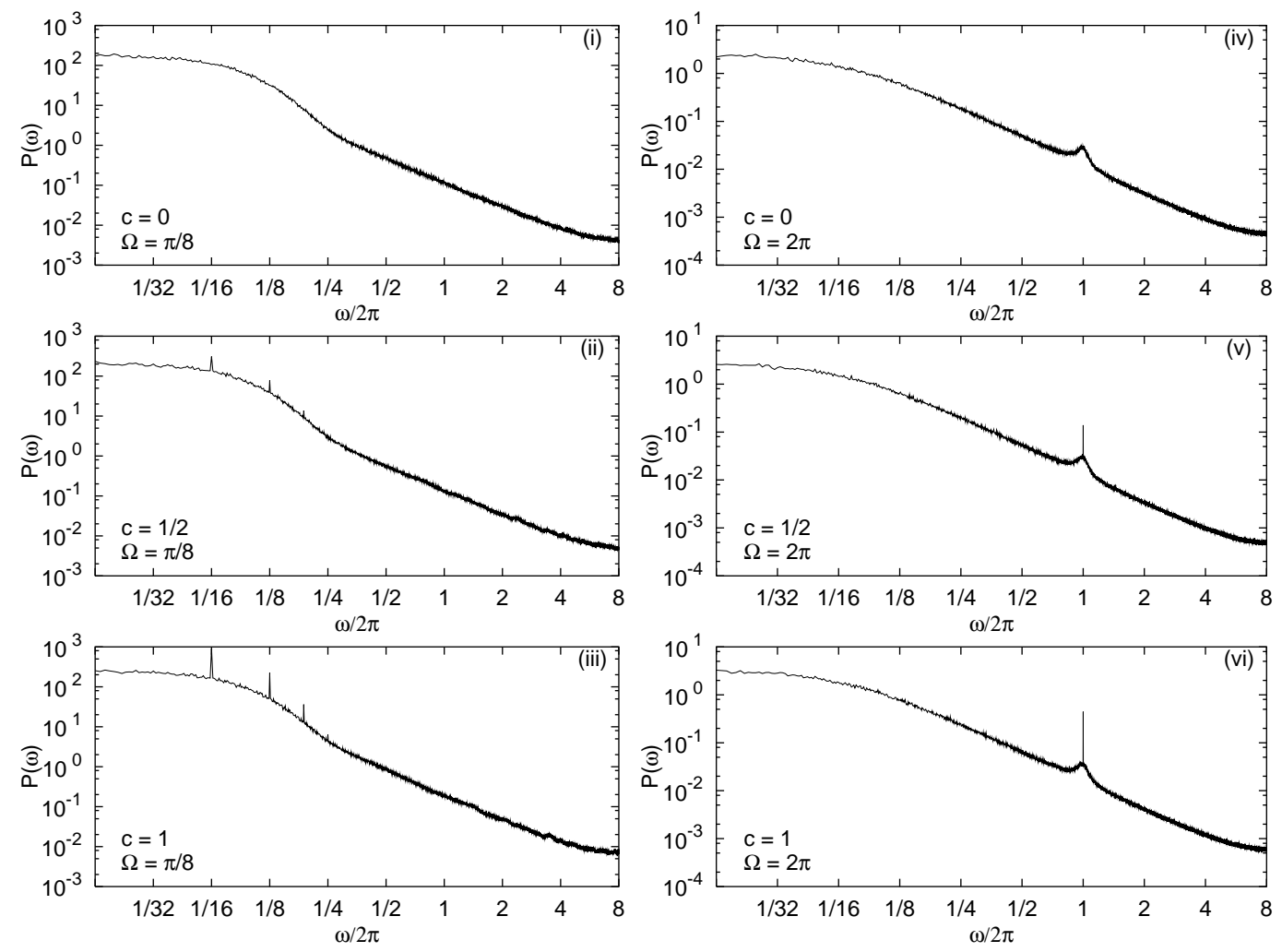

Fig. 1. Numerical power spectra of the process (22) for various input signal frequencies and correlations between the noises. The input signal frequency equals $\Omega=\pi / 8$ (panels (i) through (iii)) and $\Omega=2 \pi$ (panels (iv) through (vi)). The multiplicative and additive noises are uncorrelated ( $c=0$ ) on panels (i), (iv), partially correlated ( $c=1 / 2$ ) on panels (ii), (v), and fully correlated $(c=1)$ on panels (iii), (vi). Other parameters, common for all panels, are $k_{a}=1 / 2, \kappa=\sqrt{k_{a}} / 2, \lambda=1 / 4$, and $A=1$.

and $Z_{i}$ are certain functions involving trigonometric functions of $\tau$ and the integration variables, but not of $t$. We can see that this correlation function converges for $\kappa<\sqrt{k_{a}}=\kappa_{\max } / \sqrt{2}$. A reasoning similar to that used in (29) shows that the correlation function becomes stationary in the limit $t \rightarrow \infty$. In the limit $\Omega \rightarrow 0$, the correlation function (31) formally diverges if $A>2\left(k_{a}-\kappa_{2}\right)$. Note also that the square of the strength of the additive noise $\lambda$ multiplies all terms in (32). The situation is slightly paradoxical: without the additive noise, almost all realizations of the process $a(t)$ eventually die out, yet as in the output process the power attributed to "signal" and to "noise" scale by $\lambda^{2}$, the SNR does not depend on the additive noise level.

\subsection{Numerical results}

Because of the analytical structure of Eq. (32), any further analysis of this correlation function should be performed numerically. Instead of numerically calculat- 
ing the Fourier transform of Eq. (31), we have directly integrated Eq. (22) by the Heun method [25] with a time step $h=1 / 256$, calculated the power spectrum for each realization and averaged over 512 realizations of the noises and the initial phase. We have used Marsaglia algorithm [26] to generate the GWNs and the famous Mersenne Twister [27] as the underlying uniform generator. Selected results are presented on Fig. 1. As we can see, if the external frequency is small and the two noises are uncorrelated, the process $a(t)$ does not display any oscillations, cf. panel (i). If the noises become correlated, distinct sharp peaks corresponding to the driving frequency and its higher harmonics appear, cf. panels (ii) and (iii). For a large driving frequency, in the uncorrelated case there is a broad peak centered at the driving frequency, cf. panel (iv). If the noises get correlated, a sharp line appears, superimposed on the broad peak, cf. panels (v) and (vi).

If there are no correlations, oscillations introduced by a parametrically coupled signal are, by their very nature, damped with an effective damping constant $k_{a}-\frac{1}{2} \kappa^{2}$. If the driving frequency is very small, with $\Omega^{-1} \gg\left(k_{a}-\frac{1}{2} \kappa^{2}\right)^{-1}$, the transmitter is dominated by the damping term and the resulting power spectrum is nonoscillatory (Fig. 1, panel (i) above). For larger frequencies a broad peak, characteristic for damped oscillations, appears in the power spectrum (Fig. 1, panel (iv)). The presence of correlations between the additive and multiplicative noises changes the situation. Sharp lines, characteristic for undamped oscillations, appear in the power spectrum for both small and large driving frequencies. We can see that the correlations have a strong constructive effect on the transmitted signal in case of a multiplicative coupling.

The presence of higher harmonics of the driving frequency on panels (ii), (iii) reflects a nonlinear nature of the multiplicative coupling between the transmitter and the external signal.

\subsection{The origin of the constructive role of correlations}

To understand, at least qualitatively, why correlations between the additive and multiplicative noises reinforce the parametrically coupled signal, observe that Eq. (22) is formally equivalent to

$$
\begin{aligned}
\dot{a}= & -\left(k_{a}+\kappa \xi(t)+A \cos (\Omega t+\phi)\right)\left(a-\frac{c \lambda}{\kappa}\right) \\
& -\frac{c \lambda}{\kappa} k_{a}-\frac{c \lambda}{\kappa} A \cos (\Omega t+\phi)+\lambda \sqrt{1-c^{2}} \eta(t) .
\end{aligned}
$$


If we now introduce

$$
\widetilde{a}=a-c \lambda / \kappa
$$

we obtain

$\dot{\tilde{a}}=-\left(k_{a}+\kappa \xi(t)+A \cos (\Omega t+\phi)\right) \widetilde{a}-\frac{c \lambda}{\kappa} k_{a}-\frac{c \lambda}{\kappa} A \cos (\Omega t+\phi)+\lambda \sqrt{1-c^{2}} \eta(t)$.

The correlated part of the noise has been formally removed from Eq. (35). We can now see that the presence of correlations effectively amounts to (i) lowering of the amplitude of the additive noise, (ii) introducing a constant additive forcing, and (iii) additively coupling to the transmitter a periodic signal with exactly the same phase and frequency as the multiplicative one. The amplitude of the additional signal is proportional to the product of the correlation coefficient and the strength of the additive noise. As we already know from Section 3, an additive periodic signal introduces undamped oscillations into the correlation function which, in turn, introduce very sharp lines into the power spectrum. Such lines are clearly visible on Fig. 1.

Note that a transformation analogous to (34), when applied to the system with a purely additive signal (12), does not affect the signal. This observation, perhaps, clarifies why the presence of correlations in the additive case does not lead to any constructive effects.

\section{Discussion}

In this paper we have discussed the effects of correlations between additive and multiplicative noises in a model of a linear transmitter (5). We have shown that the effects of correlations is different depending on whether the signal is coupled additively or multiplicatively. In the former case, the correlations act destructively: The amplitude of the outgoing signal increases with the level of the multiplicative noise, but the constant shift introduced by the correlations increases even faster, the amplitude of the oscillations relative to the shift decreases, and the signal is eventually drowned by the noise. Note that the correlations act here against the noise: The multiplicative noise enhances the signal, but the correlations diminish it. Similar effects can be seen in the power spectrum and the SNR of the outgoing signal. The fact that correlations reduce the destructive effects of noise has been reported previously; papers quoted in the Introduction provide examples of such 
systems. What we have here is an example of correlations reducing the constructive effect of noise.

These formal results, and the correlation function (17) in particular, agree with those reported in Ref. [20], where the Gaussian white noise has been obtained as a limiting case of a dichotomous, colored noise. This should come as no surprise: There is a generally held belief (see e.g. [28]) that in a well defined limit the two, in principle very different, kinds of noise have identical effect on the dynamics of a system. A comparison of our present results with those of Ref. [20] provides yet another confirmation of this statement.

The situation is entirely different for the case of a multiplicative signal. We have shown that for a weak incoming signal not averaged over the phase, the outgoing signal displays oscillations that do not vanish as the level of the multiplicative noise increases. Numerical evidence suggests that without the correlations, the power spectrum averaged over both realizations of the noise and the initial phase does not display any signs of oscillations for low frequencies and only damped oscillations for larger frequencies. This is easy to understand: A multiplicative signal is, by its very nature, damped, and if the wavelength of the signal is large, the signal does not recover before if gets completely washed away by the damping. The presence of correlations changes this picture altogether: The power spectrum displays distinct, sharp lines for all frequencies of the input signal. We have showed analytically that correlations between the multiplicative and additive noises are effectively equivalent to adding an extra signal, with exactly the same phase and frequency as the multiplicative one, to the system. The amplitude of this extra signal is proportional to the correlation coefficient and it is this signal that produces the sharp lines $(\delta$ peaks) in the power spectrum.

We have assumed in Section 2 that the two noises present in the system together form a two-dimensional Gaussian process. On a formal level, this property is reflected in the relation between the amplitudes of the correlated and uncorrelated parts of the additive noise. If we relax this assumption, instead of (5) we obtain

$$
\dot{a}=-\left(k_{a}+\kappa \xi(t)+A_{\mathrm{m}} \cos (\Omega t+\phi)\right) a+\lambda_{\xi} \xi(t)+\lambda_{\eta} \eta(t)+A_{\mathrm{a}} \cos (\Omega t+\phi),
$$

where no special dependence between $\lambda_{\xi}$ and $\lambda_{\eta}$ is assumed. The results reported in this paper can now be recovered if $c^{2}$ is replaced by $\lambda_{\xi}^{2} /\left(\lambda_{\xi}^{2}+\lambda_{\eta}^{2}\right)$ and $Z=\left(\lambda / A_{\mathrm{a}}\right)^{2}$ is replaced by $\left(\lambda_{\xi}^{2}+\lambda_{\eta}^{2}\right) / A_{\mathrm{a}}^{2}$. Thus, results reported do not depend on the assumption that the joint distribution of the noises is Gaussian. They do depend, however, on the assumption that each individual noise is Gaussian and point-correlated in time.

I acknowledge a very helpful discussion with Prof. Peter Hänggi that helped me to clarify certain ambiguities present in a draft version of this paper. 


\section{A Appendix: The expectation values}

While calculating the expectation value of $a(t)$ and the correlation function of this process, one encounters several integrals that involve the expectation value of the exponential of an integral of the multiplicative noise $\xi(t)$. Specifically,

$$
\left\langle\exp \left[\int_{0}^{T} \varphi\left(t^{\prime}\right) \xi\left(t^{\prime}\right) d t^{\prime}\right]\right\rangle=\exp \left[\frac{1}{2} \int_{0}^{T}\left[\varphi\left(t^{\prime}\right)\right]^{2} d t^{\prime}\right]
$$

where $T$ is a certain time and $\varphi(t)$ is a certain function, not necessarily continuous (cf. [17,29]). Similarly,

$$
\begin{aligned}
\left\langle\eta\left(t_{1}\right) \exp \left[\int_{0}^{T} \varphi\left(t^{\prime}\right) \xi\left(t^{\prime}\right) d t^{\prime}\right]\right\rangle & =0, \\
\left\langle\eta\left(t_{1}\right) \eta\left(t_{2}\right) \exp \left[\int_{0}^{T} \varphi\left(t^{\prime}\right) \xi\left(t^{\prime}\right) d t^{\prime}\right]\right\rangle & =\delta\left(t_{1}-t_{2}\right) \exp \left[\frac{1}{2} \int_{0}^{T}\left[\varphi\left(t^{\prime}\right)\right]^{2} d t^{\prime}\right]
\end{aligned}
$$

because $\eta(t), \xi(t)$ are independent GWNs. The other two expectation values are more challenging. We start with

$$
C_{1}=\left\langle\xi\left(t_{1}\right) \exp \left[\int_{0}^{T} \varphi\left(t^{\prime}\right) \xi\left(t^{\prime}\right) d t^{\prime}\right]\right\rangle
$$

We expand the exponential function in a Taylor series, interchange the orders of summations, and obtain

$$
C_{1}=\sum_{n=0}^{\infty} \frac{1}{n !} \int_{0}^{T} d t_{\mu_{1}} \ldots \int_{0}^{T} d t_{\mu_{n}} \varphi\left(t_{\mu_{1}}\right) \ldots \varphi\left(t_{\mu_{n}}\right)\left\langle\xi\left(t_{\mu_{1}}\right) \ldots \xi\left(t_{\mu_{n}}\right) \xi\left(t_{1}\right)\right\rangle
$$

The expectation value on the right-hand side of (A.4) involves $n+1$ terms. Therefore, only the terms with $n=2 m+1$ will give a nonzero contribution to the sum. The expectation value thus involves $2(m+1)$ noise terms. It factors out to a product of $m+1$ two-point correlations and there are $(2 m+1)$ !! ways to choose the pairs. In every possible choice, one of the pairs involves $t_{1}$ and a certain $t_{\mu}$, and the remaining $m$ pairs involve two $t_{\mu}$ 's. Thus 


$$
\begin{aligned}
C_{1}=\sum_{m=0}^{\infty} \frac{(2 m+1) ! !}{(2 m+1) !}\left(\int_{0}^{T} \varphi\left(t_{\mu}\right)\left\langle\xi\left(t_{\mu}\right) \xi\left(t_{1}\right)\right\rangle d t_{\mu}\right) \times \\
\left.\qquad \int_{0}^{T} d t_{\mu_{1}} \int_{0}^{T} d t_{\mu_{2}} \varphi\left(t_{\mu_{1}}\right) \varphi\left(t_{\mu_{2}}\right)\left\langle\xi\left(t_{\mu_{1}}\right) \xi\left(t_{\mu_{2}}\right)\right\rangle\right)^{m} \\
=\sum_{m=0}^{\infty} \frac{1}{2^{m} m !} \varphi\left(t_{1}\right)\left(\int_{0}^{T}\left[\varphi\left(t_{\mu}\right)\right]^{2} d t_{\mu}\right)^{m} \\
=\varphi\left(t_{1}\right) \exp \left[\frac{1}{2} \int_{0}^{T}\left[\varphi\left(t_{\mu}\right)\right]^{2} d t_{\mu}\right] .
\end{aligned}
$$

The last remaining expectation value involves two noise terms multiplying the exponential:

$$
\begin{aligned}
C_{2} & =\left\langle\exp \left[\int_{0}^{T} \varphi\left(t^{\prime}\right) \xi\left(t^{\prime}\right) d t^{\prime}\right] \xi\left(t_{1}\right) \xi\left(t_{2}\right)\right\rangle \\
& =\sum_{n=0}^{\infty} \frac{1}{n !} \int_{0}^{T} d t_{\mu_{1}} \ldots \int_{0}^{T} d t_{\mu_{n}} \varphi\left(t_{\mu_{1}}\right) \ldots \varphi\left(t_{\mu_{n}}\right)\left\langle\xi\left(t_{\mu_{1}}\right) \ldots \xi\left(t_{\mu_{n}}\right) \xi\left(t_{1}\right) \xi\left(t_{2}\right)\right\rangle,
\end{aligned}
$$

where, as above, we have expanded the exponential into the power series. Only the terms with $n=2 m$ will give a nonzero contribution to the sum. The expectation value of the product of noise terms factors out to a product of two-point correlations. Again, there are $(2 m+1)$ !! ways to choose the pairs. In $(2 m-1)$ !! cases the noises at $t_{1}, t_{2}$ will be coupled with each other, and in the remaining cases they will be coupled with different $t_{\mu}$ s. Therefore 


$$
\begin{aligned}
& C_{2}=\sum_{m=0}^{\infty} \frac{(2 m-1) ! !}{(2 m) !}\left\langle\xi\left(t_{1}\right) \xi\left(t_{2}\right)\right\rangle \times \\
&\left(\int_{0}^{T} d t_{\mu_{1}} \int_{0}^{T} d t_{\mu_{2}} \varphi\left(t_{\mu_{1}}\right) \varphi\left(t_{\mu_{2}}\right)\left\langle\xi\left(t_{\mu_{1}}\right) \xi\left(t_{\mu_{2}}\right)\right\rangle\right)^{m} \\
&+\sum_{m=1}^{\infty} \frac{(2 m+1) ! !-(2 m-1) ! !}{(2 m) !} \times \\
& \quad\left(\int_{0}^{T} \varphi\left(t_{\mu}\right)\left\langle\xi\left(t_{\mu}\right) \xi\left(t_{1}\right)\right\rangle d t_{\mu}\right)\left(\int_{0}^{T} \varphi\left(t_{\nu}\right)\left\langle\xi\left(t_{\nu}\right) \xi\left(t_{2}\right)\right\rangle d t_{\nu}\right) \times \\
& \quad\left(\int_{0}^{T} d t_{\mu_{1}} \int_{0}^{T} d t_{\mu_{2}} \varphi\left(t_{\mu_{1}}\right) \varphi\left(t_{\mu_{2}}\right)\left\langle\xi\left(t_{\mu_{1}}\right) \xi\left(t_{\mu_{2}}\right)\right\rangle\right)^{m-1} \\
&=\delta\left(t_{1}-t_{2}\right) \sum_{m=0}^{\infty} \frac{1}{2^{m} m !}\left(\int_{0}^{T}\left[\varphi\left(t_{\mu}\right)\right]^{2} d t_{\mu}\right)^{m} \\
&+\varphi\left(t_{1}\right) \varphi\left(t_{2}\right) \sum_{m=1}^{\infty} \frac{1}{2^{m-1}(m-1) !}\left(\int_{0}^{T}\left[\varphi\left(t_{\mu}\right)\right]^{2} d t_{\mu}\right)^{m-1} \\
&\left.=\left[\delta\left(t_{1}-t_{2}\right)+\varphi\left(t_{1}\right) \varphi\left(t_{2}\right)\right] \exp \frac{1}{2} \int_{0}^{T}\left[\varphi\left(t_{\mu}\right)\right]^{2} d t_{\mu}\right]^{m}
\end{aligned}
$$

This approach can be easily generalized to colored Gaussian noises. Suppose $\xi(t)$ is a Gaussian noise with zero mean and correlations of the form

$$
\left\langle\xi(t) \xi\left(t^{\prime}\right)\right\rangle=h\left(\left|t-t^{\prime}\right|\right)
$$

where $\int_{-\infty}^{\infty} h(u) d u=1$. Then, instead of (A.7), we obtain

$$
\begin{gathered}
\left\langle\exp \left[\int_{0}^{T} \varphi\left(t^{\prime}\right) \xi\left(t^{\prime}\right) d t^{\prime}\right] \xi\left(t_{1}\right) \xi\left(t_{2}\right)\right\rangle= \\
{\left[h\left(\left|t_{1}-t_{2}\right|\right)+\left(\int_{0}^{T} \varphi\left(t_{\mu}\right) h\left(\left|t_{\mu}-t_{1}\right|\right) d t_{\mu}\right)\left(\int_{0}^{T} \varphi\left(t_{\nu}\right) h\left(\left|t_{\nu}-t_{2}\right|\right) d t_{\nu}\right)\right] \times} \\
\exp \left[\frac{1}{2} \int_{0}^{T} d t_{\mu} \int_{0}^{T} d t_{\nu} \varphi\left(t_{\mu}\right) \varphi\left(t_{\nu}\right) h\left(\left|t_{\mu}-t_{\nu}\right|\right)\right]
\end{gathered}
$$

and similar expression for other expectation values. 
As we have said, the function $\varphi$ needs not to be continuous. In many practical situations, for example in evaluating expressions like

$$
\left\langle\exp \left[\int_{t_{1}}^{t_{2}} \varphi\left(t^{\prime}\right) \xi\left(t^{\prime}\right) d t^{\prime}\right]\right\rangle
$$

with $0 \leqslant t_{1}<t_{2} \leqslant T, \varphi(t)$ would be the characteristic function of an interval:

$$
\varphi(t)= \begin{cases}0 & t<t_{1} \\ \frac{1}{2} & t=t_{1} \\ 1 & t_{1}<t<t_{2} \\ \frac{1}{2} & t=t_{2} \\ 0 & t>t_{2}\end{cases}
$$

The rationale for putting $\frac{1}{2}$ at the endpoints is that

$$
\frac{1}{2}=\int_{t_{1}}^{t_{2}} \delta\left(t-t_{1}\right) d t=\int_{-\infty}^{\infty} \varphi(t) \delta\left(t-t_{1}\right) d t=\varphi\left(t_{1}\right)
$$

The first equality stems from the fact that in $\int_{t_{1}}^{t_{2}} \delta\left(t-t_{1}\right) d t$ the integrand contains only "a half of the peak". By the same token, for example

$$
\left\langle\xi\left(t_{1}\right) \exp \left[-\kappa \int_{t_{1}}^{T} \xi\left(t^{\prime}\right) d t^{\prime}\right]\right\rangle=-\frac{1}{2} \kappa \exp \left(\frac{1}{2} \kappa^{2}\left(T-t_{1}\right)\right)
$$

where $T \geqslant t_{1}$ and Eq. (A.5) has been used.

\section{References}

[1] R. Benzi, A. Sutera, and A. Vulpiani, J. Phys. A 14 (1981) L453;

for a review see L. Gammaitoni, P. Hänggi, P. Jung, and F. Marchesoni, Rev. Mod. Phys. 70 (1998) 223.

[2] For a review see P. Reimann and P. Hänggi, Appl. Phys. A 75 (2002) 169;

for a tutorial introduction see R. D. Astumian and P. Hänggi, Phys. Today 55, No. 11 (2002) 33.

[3] S. M. Bezrukov, Phys. Lett. A 248 (1998) 29. 
[4] J. A. Freund, L. Schimansky-Geier, and P. Hänggi, Chaos 13 (2003) 225.

[5] S. M. Bezrukov and L. B. Kish, Smart Mater. Struct. 11 (2002) 800.

[6] J. Casado-Pascual, C. Denk, J. Gómez-Ordóñez, M. Morillo, and P. Hänggi, Phys. Rev. E 67 (2003) 036109.

[7] A. Fuliński and T. Telejko, Phys. Lett. A 152 (1991) 11.

[8] A. J. R. Madureira, P. Hänggi, and H. S. Wio, Phys. Lett. A 217 (1996) 248.

[9] J.-H. Li, J. Łuczka, and P. Hänggi, Phys. Rev. E 64 (2001) 011113.

[10] K. P. Singh, G. Ropars, M. Brunel, and A. Le Floch, Phys. Rev. Lett. 90 (2003) 073901.

[11] A. Fuliński and P. F. Góra, Phys. Rev. E 48 (1993) 3510.

[12] F. Liu, B. Hu, and W. Wang, Phys. Rev. E 63 (2001) 031907.

[13] C. Zhou, J. Kurths, I. Z. Kiss, and J. L. Hudson, Phys. Rev. Lett. 89 (2002) 014101;

I. Z. Kiss, Y. Zhai, J. L. Hudson, C. Zhou, and J. Kurths, Chaos 13 (2003) 267.

[14] B. Allen and J. D. Romano, Phys. Rev. D 59 (1999) 102001.

[15] Y. El-Mohri, L. E. Antonuk, Q. Zhao, M. Maolinbay, X. Rong, K.-W. Jee, S. Nassif, and C. Cionca, Med. Phys. 27 (2000) 1855.

[16] Y. Jia, J. R. Li, Physica A 252 (1998) 417.

[17] A. Fuliński, Phys. Rev. Lett. 79 (1997) 4926;

A. Fuliński and P. F. Góra, J. Stat. Phys. 101 (2000) 483.

[18] A. Fuliński and P. F. Góra, Phys. Rev. E 64 (2001) 011905.

[19] A. Fuliński, Phys. Rev. E 52 (1995) 4523;

V. Berdichevsky and M. Gitterman, Europhys. Lett. 36 (1996) 161;

L. Schimansky-Geier, T. Pöschel, and W. Ebeling, eds. Stochastic Dynamics (Springer, Berlin, 1997);

A. V. Barzykin and K. Seki, Europhys. Lett. 40 (1998) 117;

A. V. Barzykin, K. Seki, and F. Shibata, Phys. Rev. E 57 (1998) 6555;

Kwok Sau Fa, Chem. Phys. 287 (2003) 1.

[20] V. Berdichevsky and M. Gitterman, Phys. Rev. E 60 (1999) 1494.

[21] G. H. Golub and C. F. Van Loan, Matrix Computations (The John Hopkins University Press, Baltimore, 1983), chapter 4.2.3.

[22] P. Jung and P. Hänggi, Europhys. Lett. 8 (1989) 505;

P. Jung and P. Hänggi, Phys. Rev. A 44 (1991) 8032.

[23] K. Wiesenfeld, T. Wellens, and A. Buchleitner, in: A. Buchleitner and K. Hornberger, eds., Lecture Notes in Physics 611 (2002) 107.

[24] Handbook of Mathematical Functions, edited by M. Abramowitz and I. A. Stegun (Dover Publications, New York, 1968), formula 9.6.16. 
[25] R. Manella, Int. J. Mod. Phys. C 13 (2002) 1177 and references quoted therein.

[26] G. Marsaglia and T. A. Bray, SIAM Review 6 (1964) 260;

A. J. Kinderman and J. G. Ramage, J. Amer. Statist. Assoc. 71 (1976) 893;

R. Wieczorkowski and R. Zieliński, Komputerowe generatory liczb losowych (WNT, Warszawa, 1997) (in Polish).

[27] M. Matsumoto and T. Nishimura, ACM Trans. on Modeling and Computer Simulation, 8 (1998) 3.

[28] C. Van Den Broeck, J. Stat. Phys 31 (1982) 467;

A. Fuliński, Phys. Lett. A 180 (1993) 94;

A. Fuliński, Phys. Lett. A 193 (1994) 267.

[29] R. Kubo, M. Toda, and N. Hashitsume, Statistical Physics II. Nonequilibrium Statistical Mechanics (Springer, Berlin, 1985) par. 1.4. 